\title{
PSEUDOPRIME $l$-IDEALS IN A CLASS OF $f$-RINGS
}

\author{
SUZANNE LARSON
}

(Communicated by Louis J. Ratliff, Jr.)

\begin{abstract}
In a commutative $f$-ring, an $l$-ideal $I$ is called pseudoprime if $a b=0$ implies $a \in I$ or $b \in I$, and is called square dominated if for every $a \in I,|a| \leq x^{2}$ for some $x \in A$ such that $x^{2} \in I$. Several characterizations of pseudoprime $l$-ideals are given in the class of commutative semiprime $f$ rings in which minimal prime $l$-ideals are square dominated. It is shown that the hypothesis imposed on the $f$-rings, that minimal prime $l$-ideals are square dominated, cannot be omitted or generalized.
\end{abstract}

Introduction. Let $X$ be a topological space and $C(X)$ be the $f$-ring of all continuous real-valued functions on $X$ with coordinatewise operations. The following characterizations of pseudoprime $l$-ideals of $C(X)$ are known.

(L. Gillman and C. Kohls [4, 4.1]) For an l-ideal $I$ of $C(X)$, the following are equivalent:

(1) I is pseudoprime.

(2) The prime ideals containing I form a chain.

(3) $\sqrt{I}$ is prime.

In [11], Subramanian asks whether this characterization of pseudoprime $l$-ideals generalizes to semiprime $f$-rings. The answer in general is no, as can be seen by Example 2.7. In this work, we investigate pseudoprime $l$-ideals in the class of commutative semiprime $f$-rings in which minimal prime $l$-ideals are square dominated. In this class of $f$-rings, we give some alternate characterizations of pseudoprime $l$-ideals, and we show that in normal $f$-rings conditions (2) and (3) characterize pseudoprime $l$-ideals. We also show that if all prime $l$-ideals are square dominated, a generalization of condition (3) characterizes pseudoprime $l$-ideals in archimedean $f$-algebras. Finally, we show that the hypothesis imposed on our $f$-rings, that minimal prime $l$-ideals be square dominated, cannot be omitted or generalized in any way by showing that if any of the characterizations hold in a semiprime $f$-ring $A$, then all minimal prime $l$-ideals of $A$ are square dominated. We assume throughout that all rings are commutative and semiprime.

1. Preliminaries. An $f$-ring is a lattice ordered ring which is a subdirect product of totally ordered rings. For general information on $f$-rings see [2]. Given an $f$-ring $A$ and $x \in A$, we let $A^{+}=\{a \in A: a \geq 0\}, x^{+}=x \vee 0, x^{-}=(-x) \vee 0$, and $|x|=x \vee(-x)$.

Received by the editors August 21, 1987 and, in revised form, November 20, 1987. This paper was presented to the special session on Ordered Algebraic Structures of the American Mathematical Society on January 8, 1988 in Atlanta, Georgia.

1980 Mathematics Subject Classification (1985 Revision). Primary 06F25. 
A ring ideal $I$ of an $f$-ring $A$ is an $l$-ideal if $|x| \leq|y|, y \in I$ implies $x \in I$. Given any subset $S \subset A$, there is a smallest $l$-ideal containing $S$, and we denote this by $\langle S\rangle$.

Suppose $A$ is an $f$-ring and $I, J$ are $l$-ideals of $A$. We let $I: J=\{a \in A: a J \subseteq I\}$. The $l$-ideal $I$ is semiprime (prime) if $a^{2} \in I(a b \in I)$ implies $a \in I(a \in I$ or $b \in I)$. The $f$-ring $A$ is semiprime (prime) if $\{0\}$ is a semiprime (prime) $l$-ideal. We let $\sqrt{I}$ denote $\left\{a \in A: a^{n} \in I\right.$ for some $\left.n\right\}$, the smallest semiprime $l$-ideal containing $I$. In $[\mathbf{5}, 3.5]$, it is shown that:

(1.1) If $I$ is an $l$-ideal of a semiprime $f$-ring $A$, then $\bigcap_{n=1}^{\infty}\left\langle I^{n}\right\rangle$ is semiprime.

It is well known that all $l$-ideals containing a given prime $l$-ideal form a chain. The following result is also well known.

(1.2) A prime $l$-ideal $P$ of a commutative semiprime $f$-ring is minimal if and only if $a \in P$ implies there is a $b \notin P$ such that $a b=0$.

A subset $M$ of an $f$-ring $A$ is called an $m$-system if whenever $a, b \in M$ there exists an $x \in A$ such that $a x b \in M$. If in $A$, there is an $l$-ideal $I$ and an $m$-system $M$ such that $I \cap M=\varnothing$, then there is a prime $l$-ideal $P$ such that $I \subset P$, and $P \cap M=\varnothing$.

We call an ideal $I$ pseudoprime if $a b=0$ implies $a \in I$ or $b \in I$. In a semiprime $f$-ring $A$, a pseudoprime $l$-ideal contains a prime $l$-ideal as shown in $[\mathbf{1 1}, 2.1]$. Also, in a commutative $f$-ring a pseudoprime and semiprime $l$-ideal is necessarily a prime $l$-ideal as shown in $[\mathbf{8}, 4.2]$ and $[\mathbf{1 1}, 2.5]$.

An ideal $I$ of a commutative $f$-ring $A$ with identity element is a $z$-ideal if whenever $a, b \in A$, are contained in the same set of maximal ideals and $a \in I$ then $b \in I$. In $[10,2.7]$, G. Mason shows

(1.3) In a commutative $f$-ring $A$, a $z$-ideal $I$ is prime if and only if for all $a \in A$, either $a^{+} \in I$ or $a^{-} \in I$.

Henriksen, in [5] calls an $l$-ideal $I$ of an $f$-ring $A$ square dominated if $I=\{a \in$ $A:|a| \leq x^{2}$ for some $x \in A$ such that $\left.x^{2} \in I\right\}$. Every prime square dominated $l$-ideal $P$ satisfies $P=\left\langle P^{2}\right\rangle$.

An $f$-ring (and more generally a Riesz space) $A$ is called normal if $A=\left\{a^{+}\right\}^{d}+$ $\left\{a^{-}\right\}^{d}$ for all $a \in A$, or equivalently if $a \wedge b=0$ implies $A=\{a\}^{d}+\{b\}^{d}$. Several conditions equivalent to normality for an $f$-ring can be found in $[8,6.3]$, and for a Riesz space in [7, Theorem 9].

Given an $f$-ring $A$ and an element $x>0$ in $A$, the sequence $\left\{f_{n}\right\}_{n=1}^{\infty}$ is said to converge $x$-uniformly to the element $f \in A$ if for every $\varepsilon>0$, there exists a positive integer $N_{\varepsilon}$ such that $\left|f-f_{n}\right|<\varepsilon x$ for all $n \geq N_{\varepsilon}$. An $x$-uniform Cauchy sequence is defined similarly. If for every $x \geq 0$, every $x$-uniform Cauchy sequence has a unique limit, then $A$ is said to be uniformly complete. We say $A$ is archimedean if $a, b \in A^{+}$with $n a \leq b$ for all $n$ implies $a=0$. If $A$ is an archimedean $f$-algebra with identity element, it is well known that $A$ is commutative and semiprime. In $[1,4.1(\mathrm{~d})]$, it is shown that:

(1.4) For any archimedean $f$-algebra $A$ with identity element, there is an embedding $e$ of $A$ into a uniformly complete $f$-algebra $A^{*}$ (the uniform completion of $A)$.

2. In this section we give several characterizations of pseudoprime $l$-ideals in the class of commutative semiprime $f$-rings with minimal prime $l$-ideals square dominated. This class contains, of course, all commutative semiprime square root 
closed $f$-rings. First we give a lemma that will be used later and that also gives a characterization of commutative semiprime $f$-rings in which minimal prime $l$-ideals are square dominated.

LEMMA 2.1. Let $A$ be a commutative semiprime $f$-ring.

(1) A semiprime l-ideal $I$ is square dominated if every prime l-ideal minimal with respect to containing $I$ is square dominated.

(2) Every minimal prime l-ideal of $A$ is square dominated if and only if for every $a \in A^{+}$, the l-ideal $\{a\}^{d}=\{b \in A: a b=0\}$ is square dominated.

Proof. (1) Let $b \in I^{+}$. Let $M=\left\{c_{1}^{2} \cdots c_{n}^{2}: n \in \mathbf{N} ; b \leq c_{i}^{2}\right\}$. Then $M$ is an $m$ system. Suppose that $M \cap I=\varnothing$. Then there is a prime $l$-ideal $P$ such that $I \subseteq P$ and $M \cap P=\varnothing$. Let $P_{1} \subseteq P$ be a prime $l$-ideal minimal with respect to containing I. By hypothesis, $P_{1}$ is square dominated, so there is a $p \in A$ such that $b \leq p^{2}$ and $p^{2} \in P_{1}$. But then $p^{2} \in M \cap P$, contrary to assumption. So $M \cap I \neq \varnothing$. Let $c_{1}^{2} \cdots c_{n}^{2} \in M \cap I$, where $b \leq c_{i}^{2}$ for each $i$. Then $b \leq c_{1}^{2} \wedge \cdots \wedge c_{n}^{2}=\left(\left|c_{1}\right| \wedge \cdots \wedge\left|c_{n}\right|\right)^{2}$. Also, $0 \leq\left(\left|c_{1}\right| \wedge \cdots \wedge\left|c_{n}\right|\right)^{2 n} \leq c_{1}^{2} \cdots c_{n}^{2}$. This implies $\left(\left|c_{1}\right| \wedge \cdots \wedge\left|c_{n}\right|\right)^{2 n} \in I$, and because $I$ is semiprime, $\left(\left|c_{1}\right| \wedge \cdots \wedge\left|c_{n}\right|\right)^{2} \in I$.

$(2) \Rightarrow$ Suppose that every minimal prime $l$-ideal is square dominated. Let $a \in$ $A^{+}$. Suppose $P$ is a prime $l$-ideal minimal with respect to containing $\{a\}^{d}$. Then $M=\{b: b \in A \backslash P\} \cup\left\{a^{n}: n \in \mathbf{N}\right\} \cup\left\{b a^{n}: b \in A \backslash P, n \in \mathbf{N}\right\}$ is an $m$-system such that $M \cap\{a\}^{d}=\varnothing$. So there is a prime $l$-ideal $P_{1}$ satisfying $\{a\}^{d} \subseteq P_{1} \subseteq P$. But our choice of $P$ implies $P_{1}=P$ and $a \notin P$.

Now if $P_{2}$ is a minimal prime $l$-ideal contained in $P, a \notin P_{2}$ implies $\{a\}^{d} \subseteq P_{2}$. Hence $P_{2}=P$, and $P$ is in fact a minimal prime $l$-ideal which is square dominated. So every prime $l$-ideal minimal with respect to containing $\{a\}^{d}$ is square dominated, and part (1) implies $\{a\}^{d}$ is square dominated.

$\Leftarrow$ Let $P$ be a minimal prime $l$-ideal, and $f \in P$. By 1.2 , there is a $g \notin P$ such that $f g=0$. By hypothesis, $\{g\}^{d}$ is square dominated. So there is $f_{1} \in\{g\}^{d}$ such that $f \leq f_{1}^{2}$ and $f_{1}^{2} \in P$.

Our first characterization of pseudoprime $l$-ideals follows.

THEOREM 2.2. Let $A$ be a commutative, semiprime $f$-ring with identity element and in which every minimal prime l-ideal is square dominated. The following are equivalent for an l-ideal I:

(1) $I$ is pseudoprime.

(2) $\bigcap_{n=1}^{\infty}\left\langle I^{n}\right\rangle$ is prime.

(3) $\langle I \sqrt{I}\rangle$ is pseudoprime.

(4) $I: \sqrt{I}$ is pseudoprime and $I: \sqrt{I} \subseteq \sqrt{I}$, or, $\sqrt{I} \subseteq I: \sqrt{I}$ and $\sqrt{I}$ is prime.

PROOF. (1) $\Rightarrow(2)$. Let $P$ be a minimal prime $l$-ideal contained in $I$. Since $P$ is square dominated, $P=\bigcap_{n=1}^{\infty}\left\langle P^{n}\right\rangle \subseteq \bigcap_{n=1}^{\infty}\left\langle I^{n}\right\rangle$. So $\bigcap_{n=1}^{\infty}\left\langle I^{n}\right\rangle$ is pseudoprime. By 1.1 , it is also semiprime and therefore prime.

(2) $\Rightarrow(3)$. Trivial.

$(3) \Rightarrow(4)$. By (3), all $l$-ideals containing $\langle I \sqrt{I}\rangle$ form a chain. So $I: \sqrt{I} \subseteq \sqrt{I}$ or $\sqrt{I} \subseteq I: \sqrt{I}$.

$(4) \Rightarrow(1)$. Either hypothesis implies that there is a minimal prime $l$-ideal $P$ contained in $\sqrt{I}$ and also in $I: \sqrt{I}$. Then since $P$ is square dominated, $P=\left\langle P^{2}\right\rangle \subseteq$ $(I: \sqrt{I}) \sqrt{I} \subseteq I$. 
Recall that in a commutative ring, an ideal $I$ is primary if $a b \in I, a \notin I$ implies $b^{n} \in I$ for some $n$. It is well known that $\sqrt{I}$ is prime for every primary $l$-ideal $I$. Thus in $C(X)$, every primary $l$-ideal is pseudoprime. This is not true in general (as can be seen by Example 2.7) and the following corollary gives a condition under which primary $l$-ideals are pseudoprime.

COROLlaRY 2.3. Let $A$ be a commutative semiprime $f$-ring with identity element in which every minimal prime l-ideal is square dominated. A primary l-ideal $I$ is pseudoprime if and only if $I: \sqrt{I}$ is pseudoprime.

ProOF. Assume that $I: \sqrt{I}$ is pseudoprime. If $I=\sqrt{I}$, then $I$ is prime, so we may assume that $I \neq \sqrt{I}$. Let $a \in \sqrt{I} \backslash I$. We will show $I: \sqrt{I} \subseteq \sqrt{I}$. Suppose $b \in I: \sqrt{I}$. Then $a b \in I$, and since $a \notin I$, we must have $b \in \sqrt{I}$. So $I: \sqrt{I} \subseteq \sqrt{I}$. It follows from the previous theorem that $I$ is pseudoprime.

It is well known that in $C(X)$, an $l$-ideal $I$ is pseudoprime if and only if $\sqrt{I}$ is prime [4, 4.1]. In [11], Subramanian asks whether this characterization of pseudoprime $l$-ideals holds in semiprime $f$-rings. The answer in general is no (even in archimedean $f$-rings), as witnessed by Example 2.7. However, our next goal is to show that the characterization of pseudoprime $l$-ideals as those $l$-ideals $I$ for which $\sqrt{I}$ is prime also holds in a class of normal $f$-rings.

First, we will give two characterizations of normal $f$-rings, one of which is the $f$-ring analogue to a characterization given by Huijsmans in [7]. It should also be noted that the $f$-ring $C(X)$ is normal if and only if the topological space $X$ is an $F$-space and that the characterizations of normal $f$-rings given next are similar to two characterizations of $F$-spaces given in [3,14.25]. If $P$ is any prime ideal, the $P$ component of 0 is $O_{P}=\{a \in A: \exists b \notin P$ such that $a b=0\}$.

THEOREM 2.4. Let $A$ be a commutative semiprime $f$-ring with identity element. The following are equivalent.

(1) $A$ is normal.

(2) Every ideal $O_{P}$, where $P$ is a (proper) prime l-ideal, is prime.

(3) Every ideal $O_{M}$, where $M$ is a maximal l-ideal, is prime.

ProOF. (1) $\Rightarrow(2)$. Since $O_{P}$ is a $z$-ideal, 1.3 implies that it will suffice to show that for all $a \in A$, either $a^{+} \in O_{P}$ or $a^{-} \in O_{P}$. Suppose $a \in A$. If $a^{+} \notin O_{P}$ and $a^{-} \notin O_{P}$, then $\left\{a^{+}\right\}^{d} \subseteq P$ and $\left\{a^{-}\right\}^{d} \subseteq P$. But this would imply $A=\left\{a^{+}\right\}^{d}+\left\{a^{-}\right\}^{d} \subseteq P$, contrary to hypothesis.

(2) $\Rightarrow(3)$. Obvious.

(3) $\Rightarrow(1)$. Suppose $A$ is not normal. Then there is an element $a \in A$ such that $\left\{a^{+}\right\}^{d}+\left\{a^{-}\right\}^{d} \neq A$. Let $M$ be a maximal $l$-ideal containing the $l$-ideal $\left\{a^{+}\right\}^{d}+\left\{a^{-}\right\}^{d}$. Then $O_{M}$ is a $z$-ideal such that $a^{+} \notin O_{M}$ and $a^{-} \notin O_{M}$. By 1.3, $O_{M}$ is not prime, contrary to our hypothesis.

This characterization allows us to make the following observation.

LEMMA 2.5. If $A$ is a commutative, semiprime normal $f$-ring with identity element then every minimal prime l-ideal of $A$ is square dominated.

ProOF. Let $P$ be a minimal prime $l$-ideal of $A$. Since the $l$-ideals containing $P$ form a chain, there is a unique maximal $l$-ideal $M$ containing $P$. Now $O_{M} \subseteq P$ and, by the previous theorem $O_{M}$ is prime. Therefore, $O_{M}=P$. We will show $O_{M}$ 
is square dominated. Let $a \in O_{M}^{+}$. Then there exists a $b>0$ such that $b \notin M$ and $a b=0$. Then $a \wedge b=0$ and $\{a\}^{d}+\{b\}^{d}=A$. Thus, there exists $x \in\{a\}^{d}, y \in\{b\}^{d}$ such that $x+y=a \vee 1$. It follows from $y \in\{b\}^{d}$ and $b \notin M$, that $y \in O_{M}$. We have $y \in O_{M}$, and $a \leq y^{2}$.

In $[9,3.6]$ it is shown that if $I$ is an $l$-ideal of a commutative semiprime $f$-ring with identity element such that $I=I: \sqrt{I}$, then $I$ is an intersection of primary $l$-ideals. We will use this fact in the proof of the next theorem.

THEOREM 2.6. Let $A$ be a commutative semiprime normal $f$-ring with identity element. The following are equivalent for an l-ideal $I$.

(1) I is pseudoprime.

(2) The prime l-ideals containing I form a chain.

(3) $\sqrt{I}$ is prime.

ProOF. We need only show $(3) \Rightarrow(1)$. Suppose that $\sqrt{I}$ is prime. Let $P$ be a minimal prime $l$-ideal contained in $\sqrt{I}$ and $J=P \cap I$. We will show that $J$ is pseudoprime. Knowing that $\sqrt{J}$ is square dominated (Lemma 2.5), it is not hard to show that $J: \sqrt{J}=(J: \sqrt{J}): \sqrt{J: \sqrt{J}}$. Let $M$ be the maximal $l$-ideal containing $P$. For any $x \in M \backslash J: \sqrt{J}$, there is a primary $l$-ideal $Q_{x}$ containing $J: \sqrt{J}$, but not $x$ by the result mentioned above $[9,3.6]$. The $l$-ideals containing $P$ form a chain, so $P=\sqrt{J} \subseteq \sqrt{J: \sqrt{J}} \subseteq \sqrt{Q_{x}} \subseteq M$. Knowing $Q_{x} \subseteq M$, it is easy to show that $O_{M} \subseteq Q_{x}$ for all $x$. Now $J: \sqrt{J}=M \cap\left(\cap Q_{x}\right)$, so $O_{M} \subseteq J: \sqrt{J}$. By Theorem 2.4, $O_{M}$ is a prime $l$-ideal. Thus $J: \sqrt{J}$ is a pseudoprime $l$-ideal. Also, since the $l$-ideals containing $O_{M}$ form a chain, we have $J: \sqrt{J} \subseteq \sqrt{J}$ or $\sqrt{J} \subseteq J: \sqrt{J}$. In either case, Theorem 2.1 now implies that $J$ is pseudoprime.

In particular, this result implies that in an $f$-ring satisfying the hypotheses of the theorem, every primary $l$-ideal is pseudoprime.

The next example shows that the hypothesis of normality cannot be left out of this theorem. It also shows that the characterization of pseudoprime $l$-ideals as being those $I$ for which $\sqrt{I}$ is prime does not hold in archimedean $f$-algebras. In fact, primary $l$-ideals in archimedean $f$-algebras are not necessarily pseudoprime.

EXAMPLE 2.7. Let $B=\left\{f \in C[0,1]: \exists x_{f} \in(0,1)\right.$ such that $f(x)=\sum_{i=1}^{n} a_{i} x^{r_{i}}$, where $a_{i} \in \mathbf{R}, r_{i} \in \mathbf{Q}$ for all $\left.x \in\left[0, x_{f}\right]\right\}$. Let $P=\{f \in B: f(0)=0\}$. Then $P$ is a prime $l$-ideal of $B$. Let $A=\{(f, g) \in B \times B: f-g \in P\}$. Then as shown in [6], $A$ is a semiprime archimedean $f$-algebra with identity element. It is not hard to show that every prime $l$-ideal of $A$ is square dominated.

Let $I=\left\{(f, g) \in A: f(x), g(x) \leq n x^{2}\right.$ for some $\left.n, \forall x \in\left[0, x_{f} \wedge x_{g}\right]\right\}$. Then $I$ is an $l$-ideal of $A$. We will show that $I$ is primary. Suppose $(f, g)(h, k) \in I$, and $(f, g) \notin I$. For every $n$, either $f(x) \npreceq n x^{2}$ or $g(x) \$ n x^{2}$ on $[0, a)$ for any $a \in(0,1)$. Suppose $f(x) \npreceq n x^{2}$. Then $h(0)=0$ and $h \in P$. But $h-k \in P$, so $k \in P$. This implies there must exist some $N$ such that $(h, k)^{N} \in I$. Hence $I$ is primary, and $\sqrt{I}$ is prime. Yet $I$ is not pseudoprime since $(x, 0)(0, x)=(0,0)$ while $(x, 0) \notin I$ and $(0, x) \notin I$.

To see directly that $A$ is not normal, consider the element $(x,-x):\left\{(x,-x)^{+}\right\}^{d}+$ $\left\{(x,-x)^{-}\right\}^{d}=\{(x, 0)\}^{d}+\{(0, x)\}^{d}=\{(f, g): f, g \in P\} \neq A$. 
We have found some generalizations of the condition that $\sqrt{I}$ be prime that characterize pseudoprime $l$-ideals in archimedean $f$-algebras in which minimal prime $l$ ideals are square dominated. However, each of these conditions are difficult to verify for most $l$-ideals. If we strengthen our hypotheses to insist that all prime $l$-ideals of the $f$-algebra are square dominated, we can give the following generalization to the condition which is a characterization of pseudoprime $l$-ideals.

THEOREM 2.8. Let $A$ be an archimedean $f$-algebra with identity element in which prime l-ideals are square dominated. If $I$ is an l-ideal then $I$ is pseudoprime if and only if when $\left\{f_{1 j}\right\}_{j=1}^{\infty}, \ldots,\left\{f_{n j}\right\}_{j=1}^{\infty}$ are increasing positive Cauchy sequences such that $f_{1 j} f_{2 j} \cdots f_{n j}=0$ for all $j$, there is a sequence $\left\{f_{l j}\right\}_{j=1}^{\infty}$, for which there exists a positive integer $N$ such that $f_{l j}^{N} \in I$ for all $j$.

Proof. Let $A^{*}$ denote the uniform completion of $A$, and $e: A \rightarrow A^{*}$ be the embedding.

$\Leftarrow$ Suppose that whenever $\left\{f_{1 j}\right\}_{j=1}^{\infty}, \ldots,\left\{f_{n j}\right\}_{j=1}^{\infty}$ are increasing positive Cauchy sequences such that $f_{1 j} f_{2 j} \cdots f_{n j}=0$ for all $j$, there is a sequence $\left\{f_{l j}\right\}_{j=1}^{\infty}$, for which there exists a positive integer $N$ such that $f_{l j}^{N} \in I$ for all $j$. In $A^{*}$, let $M=\left\{x_{1} \cdots x_{m}\right.$ : for each $x_{i}, \forall j \exists a_{i j} \in A$ such that $0 \leq e\left(a_{i j}\right) \leq x_{i}^{j}$ and $\left.a_{i j}^{j} \notin I\right\}$. Then $M$ is an $m$-system. First we will show that $M \cap\{0\}=\varnothing$. Suppose that for $i=1,2, \ldots, m, x_{i} \in A^{*}$, such that for every $j \in \mathbf{N}$, there is an $a_{i j} \in A$ such that $0 \leq e\left(a_{i j}\right) \leq x_{i}^{j}$ and $a_{i j}^{j} \notin I$. Define

$$
f_{i j}=\sum_{k=1}^{j} \frac{1}{2^{k}}\left(a_{i k} \wedge 1\right) \quad \text { for } i=1,2, \ldots, m, j=1,2, \ldots
$$

Then $0 \leq\left(1 / 2^{j}\right) e\left(a_{i j} \wedge 1\right) \leq e\left(f_{i j}\right) \leq x_{i}$, for $i=1,2, \ldots, m, j=1,2, \ldots$, and $\left\{f_{i j}\right\}_{j=1}^{\infty}$ is an increasing positive Cauchy sequence for $i=1,2, \ldots, m$. Now for each sequence $\left\{f_{i j}\right\}_{j=1}^{\infty}$, there cannot be a natural number $N$ such that $f_{i j}^{N} \in I$ for all $j$, because the existence of such an $N$ would imply that $\left(a_{i j} \wedge 1\right)^{N}$, and hence $a_{i j}^{N}$, is in $I$ for all $j$. So by hypothesis, $f_{1 j} f_{2 j} \cdots f_{m j} \neq 0$ for some $j$. This, and the fact that $0 \leq e\left(f_{1 j}\right) e\left(f_{2 j}\right) \cdots e\left(f_{m j}\right) \leq x_{1} x_{2} \cdots x_{m}$ implies $x_{1} x_{2} \cdots x_{m} \neq 0$. Therefore, $M \cap\{0\}=\varnothing$.

Thus there is a prime $l$-ideal in $A^{*}$ containing $\{0\}$ and disjoint from $M$. Let $P$ be a minimal prime $l$-ideal with this property. We will show $P \cap e(A) \subseteq e(I)$. Let $f \in A^{+}$such that $e(f) \in P \cap e(A)$.

By 1.2 , there is a $g \notin P$ such that $e(f) g=0$. In $A,\{g\}_{A}^{d}=\{x \in A: x g=0\}$ is a semiprime $l$-ideal and Lemma 2.1(1) implies it is square dominated. So $f \leq f_{1}^{2}$ for some $f_{1} \in A^{+}$with $f_{1}^{2} \in\{g\}_{A}^{d}$. Since $\{g\}_{A}^{d}$ is semiprime, $f_{1} \in\{g\}_{A}^{d}$. As before, $f_{1} \leq f_{2}^{2}$ for some $f_{2} \in A^{+}$with $f_{2}^{2} \in\{g\}_{A}^{d}$. Again $f_{2} \in\{g\}_{A}^{d}$. So $f \leq f_{1}^{2} \leq f_{2}^{4}$. Continuing this for $i=3,4, \ldots$, we find $f \leq f_{1}^{2} \leq f_{2}^{4} \leq \cdots \leq f_{i}^{2^{i}} \leq \cdots$. Define

$$
h_{j}=\sum_{i=1}^{j} \frac{1}{2^{i}}\left(f_{i} \wedge 1\right) \quad \text { for } j=1,2, \ldots
$$

Then $\left\{h_{j}\right\}_{m=1}^{\infty}$ is an increasing Cauchy sequence in $A$ and $\left\{e\left(h_{j}\right)\right\}_{j=1}^{\infty}$ converges to an element $h \in A^{*}$. Now $h g=0$ and $g \notin P$, implying $h \in P$. 
We assert that there exists some positive integer $M$ such that

$$
\left(1 / 2^{M}\right)\left(f_{M} \wedge 1\right)^{2^{M}} \in I .
$$

For if not, $0 \leq\left(1 / 2^{i}\right)\left(f_{i} \wedge 1\right)^{2^{i}} \leq\left(f_{i} \wedge 1\right)^{i^{2}}$ would imply $\left(f_{i} \wedge 1\right)^{i^{2}}=\left(\left(f_{i} \wedge 1\right)^{i}\right)^{i} \notin I$ for all $i \neq 3$. We would then have

$$
0 \leq\left(1 / 2^{i^{2}}\right)\left(f_{i} \wedge 1\right)^{i} \leq h^{i} \text { and }\left(\left(1 / 2^{i^{2}}\right)\left(f_{i} \wedge 1\right)^{i}\right)^{i} \notin I \quad \text { for all } i .
$$

But this would imply $h \in M$, contrary to the fact that $M \cap P=\varnothing$. So there exists some positive integer $M$ such that $\left(1 / 2^{M}\right)\left(f_{M} \wedge 1\right)^{2^{M}} \in I$.

We now know $\left(1 / 2^{M}\right)(f \wedge 1) \leq\left(1 / 2^{M}\right)\left(f_{M} \wedge 1\right)^{2^{M}}$ implies $\left(1 / 2^{M}\right)(f \wedge 1) \in I$. Therefore $(f \wedge 1) \in I$ and $f=(f \wedge 1)(f \vee 1) \in I$. We have $P \cap e(A) \subseteq e(I)$ and $P \cap e(A)$ is prime in $e(A)$. Therefore $e(I)$ is pseudoprime in $A^{*}$. This implies $I$ is pseudoprime in $A$.

$\Rightarrow$ Suppose that $I$ is pseudoprime and that $\left\{f_{1 j}\right\}_{j=1}^{\infty}, \ldots,\left\{f_{n j}\right\}_{j=1}^{\infty}$ are increasing positive Cauchy sequences in $A$ with $f_{1 j} f_{2 j} \cdots f_{n j}=0$ for all $j$. Each of the sequences $\left\{e\left(f_{i j}\right)\right\}_{j=1}^{\infty}$ converges to some element $f_{i}$ in $A^{*}$. Let $P$ be a prime $l$ ideal contained in $I$. Then $M=\{e(a): a \in A, a \notin P\}$ is an $m$-system in $A^{*}$. So there is a prime $l$-ideal $P^{*}$ of $A^{*}$ such that $P \subseteq P^{*}$ and $P^{*} \cap M=\varnothing$. Now $e\left(f_{1}\right) e\left(f_{2}\right) \cdots e\left(f_{n}\right)=0$ and so $e\left(f_{m}\right) \in P^{*}$ for some $m$. Thus $e\left(f_{m j}\right) \leq e\left(f_{m}\right)$ implies that $e\left(f_{m j}\right) \in P^{*} \cap e(A)=e(P)$ for all $j$.

It is not difficult to show directly that in a uniformly complete $f$-ring, the property characterizing a pseudoprime $l$-ideal $I$ given in this theorem is equivalent to the property that $\sqrt{I}$ is prime.

Finally we show that the hypothesis that minimal prime $l$-ideals be square dominated cannot be dropped or generalized in any way in any of our theorems characterizing pseudoprime $l$-ideals.

LEMMA 2.9. Let $A$ be a semiprime f-ring. If in A, a pseudoprime l-ideal $I$ is characterized by being an l-ideal that satisfies any one of the following conditions, then every minimal prime l-ideal of $A$ is square dominated.

(1) $\bigcap_{n=1}^{\infty}\left\langle I^{n}\right\rangle$ is prime.

(2) $\langle I \sqrt{I}\rangle$ is pseudoprime.

(3) $I: \sqrt{I}$ is pseudoprime and $I: \sqrt{I} \subseteq \sqrt{I}$, or, $\sqrt{I} \subseteq I: \sqrt{I}$ and $\sqrt{I}$ is prime.

(4) The prime l-ideals containing I form a chain.

(5) $\sqrt{I}$ is prime.

Also, if $A$ is an archimedean $f$-ring, and if in $A$, a pseudoprime l-ideal $I$ is characterized by being an l-ideal that satisfies the following condition, then every minimal prime $l$-ideal of $A$ is square dominated.

(6) Whenever $\left\{f_{1 j}\right\}_{j=1}^{\infty}, \ldots,\left\{f_{n j}\right\}_{j=1}^{\infty}$ are increasing positive Cauchy sequences such that $f_{1 j} f_{2 j} \cdots f_{n j}=0$ for all $j$, there is a sequence $\left\{f_{l j}\right\}_{j=1}^{\infty}$, for which there exists a positive integer $N$ such that $f_{l j}^{N} \in I$ for all $j$.

Proof. Let $P$ be a minimal prime $l$-ideal. Note that in each case it will suffice to show that $\left\langle P^{2}\right\rangle$ is pseudoprime since $\left\langle P^{2}\right\rangle \subseteq P$ and $P$ being a minimal prime $l$-ideal will then imply $\left\langle P^{2}\right\rangle=P$.

If characterization (1) holds, then $\bigcap_{n=1}^{\infty}\left\langle P^{n}\right\rangle$ is a prime $l$-ideal contained in $\left\langle P^{2}\right\rangle$. So $\left\langle P^{2}\right\rangle$ is pseudoprime. 
Characterization (2) implies $\langle P \sqrt{P}\rangle=\left\langle P^{2}\right\rangle$ is pseudoprime.

Suppose characterization (3) holds. Note that $\left\langle P^{2}\right\rangle: \sqrt{\left\langle P^{2}\right\rangle}=\left\langle P^{2}\right\rangle: P \supseteq$ $\sqrt{\left\langle P^{2}\right\rangle}=P$. So $\left\langle P^{2}\right\rangle: \sqrt{\left\langle P^{2}\right\rangle}$ is pseudoprime. Then characterization (3) implies $\left\langle P^{2}\right\rangle$ is pseudoprime.

Suppose characterization (4) holds. Every prime $l$-ideal containing $\left\langle P^{2}\right\rangle$ also contains $P$. So the prime $l$-ideals containing $\left\langle P^{2}\right\rangle$ form a chain. Characterization (4) implies $\left\langle P^{2}\right\rangle$ is pseudoprime.

Suppose characterization (5) holds. Then $\sqrt{\left\langle P^{2}\right\rangle}=P$ is prime, implying that $\left\langle P^{2}\right\rangle$ is pseudoprime.

Finally, suppose that $A$ is an archimedean $f$-algebra and that characterization (6) holds. Suppose that $\left\{f_{1 j}\right\}_{j=1}^{\infty}, \ldots,\left\{f_{n j}\right\}_{j=1}^{\infty}$ are increasing positive Cauchy sequences such that $f_{1 j} f_{2 j} \cdots f_{n j}=0$ for all $j$. Then for some $m, f_{m j} \in P$ for all $j$. But then $f_{m j}^{2} \in\left\langle P^{2}\right\rangle$ for all $j$. So characterization (6) implies that $\left\langle P^{2}\right\rangle$ is pseudoprime.

\section{REFERENCES}

1. E. Aron and A. Hager, Convex vector lattices and l-algebras, Topology Appl. 12 (1981), 1-10.

2. A. Bigard, K. Keimel and S. Wolfenstein, Groups et anneaux reticules, Lecture Notes in Math., vol. 608, Springer-Verlag, 1977.

3. L. Gillman and M. Jerison, Rings of continuous functions, Springer-Verlag, 1960.

4. L. Gillman and C. Kohls, Convex and pseudoprime ideals in rings of continuous functions, Math. Z. 72 (1960), 399-409.

5. M. Henriksen, Semiprime ideals of $f$-rings, Sympos. Math. 21 (1977), 401-409.

6. M. Henriksen and F. A. Smith, Sums of $z$-ideals and semiprime ideals, General Topology and Its Relations to Modern Analysis and Algebra 5 (1982), 272-278.

7. C. B. Huijsmans, Some analogies between commutative rings, Riesz spaces and distributive lattices with smallest element, Proc. Nederl. Akad. Wetensch. A77=Indag. Math. 36 (1974), 132-147.

8. C. B. Huijsmans and B. de Pagter, Ideal theory in $f$-algebras, Trans. Amer. Math. Soc. 269 (1982), 225-245.

9. S. Larson, Minimal convex extensions and intersections of primary l-ideals in $f$-rings, J. Algebra (to appear).

10. G. Mason, $z$-ideals and prime ideals, J. Algebra 26 (1973), 280-297.

11. H. Subramanian, l-prime ideals in f-rings, Bull. Soc. Math. France 95 (1967), 193-203.

Department of Mathematics, loyola Marymount University, Los Angeles, CALIFORNIA 90045 